\title{
Fibróma traumático en cavidad oral - una revisión
}

\author{
Traumatic fibroma - a review
}

\section{Resumen}

\author{
Rocafuerte - Acurio Marco Antonio ${ }^{1 a}$
}

El crecimiento fibroso intraoral de los tejidos blandos es relativamente común y puede ser de carácter benigno reactivo o una lesión neoplásica. Entre estos crecimientos fibrosos localizados, se ha encontrado que el fibroma traumático ocurre comúnmente en la cavidad oral. El fibroma traumático es una hiperplasia fibrosa reactiva causada por un traumatismo o irritación local como puede ser la presencia de cálculo, los márgenes sobresalientes de las restauraciones, aparatología ortodóntica, entre otros. Esta lesión también es conocida con los nombres de fibroma por irritación, fibroma inflamatorio, hiperplasia fibrosa focal, nódulo fibroso, pólipo fibroso. Se puede presentar en cualquier sitio de la cavidad oral pero se observa con mayor frecuencia en la mucosa bucal o a lo largo del plano oclusal. El fibroma traumático es una lesión frecuente en la cavidad oral por lo que un amplio conocimiento sobre esta lesión sería útil para los odontólogos en el tratamiento de sus pacientes.

Palabras clave: Fibroma traumático; Hiperplasia; Etiología; Prevalencia. (Fuente: DeCS BIREME)

\section{ABSTRACT}

The intraoral fibrous growth of the soft tissues is relatively common and may be benign reactive or a neoplastic lesion. Among these localized fibrous growths, it has been found that traumatic fibroma is the most common in the oral cavity. The traumatic fibroma is a reactive fibrous hyperplasia caused by trauma or local irritation, such as the presence of calculus, overhanging margins of restorations, orthodontic appliances, among others. This pathology is also known as irritation fibroma, inflammatory fibroma, focal fibrous hyperplasia, fibrous nodule and fibrous polyp. It can occur anywhere in the oral cavity but it is more frequently seen in the buccal mucosa or along the occlusal plane. Traumatic fibroma is a common lesion in the oral cavity. Therefore, a wide knowledge about this injury would be useful for dentists in the treatment of their patients.

Key words: Traumatic fibroma; Hyperplasia; Etiology; Prevalence. (Source: MeSH NLM)

Recibido: 15 de noviembre de 2018 1 Universidad Complutense de Madrid (UCM). Madrid, España

a Especialista en Medicina Oral

Correspondencia:

Marco Antonio Rocafuerte Acurio

Correo eelctronico:mrocafuertea@gmail.com
Aprobado: 25 de enero de 2018

Publicado: 30 de enero de 2019

Este es un artículo Open Access distribuido bajo ta licencia Creative Commons Atribución-NoComercialCompartir Igual 4.0

\section{ㄷ)(1)(2)(2)}




\section{INTRODUCCIÓN}

Las lesiones más comunes en la cavidad oral son crecimientos fibrosos hiperplásicos focales localizados los que incluyen la hiperplasia fibrosa focal, granuloma piógeno, fibroma traumático, fibroma osificante periférico y granuloma de células gigantes (1). Entre estos agrandamientos fibrosos localizados, se ha encontrado que el fibroma traumático es el más común en la cavidad oral (2).

El fibroma traumático es una hiperplasia fibrosa focal reactiva causada por traumatismo o irritación local. Esto puede deberse a irritantes como: cálculo, placa, márgenes sobresalientes de las restauraciones, aparatología ortodóntica, etc ${ }^{(3)}$.

Es un tumor benigno de los tejidos blandos de crecimiento lento. Suelen ser masas sésiles, pedunculadas y asintomáticas. Se le conoce también con los nombres de fibroma de irritación, fibroma inflamatorio, hiperplasia fibrosa focal, nódulo fibroso y pólipo fibroso. El trauma localizado puede ocurrir como episodio único o episodios repetidos siendo estos menos graves ${ }^{(4)}$.

La infección crónica también puede causar un fibroma traumático. Existe otro tipo fibroma, el cual se llama fibroma verdadero, pero este ocurre raramente en áreas orales y maxilofaciles. El fibroma verdadero es un crecimiento que no necesariamente se origina con un potencial trauma ${ }^{(5)}$.

En el año 1967, Barquer y Lucas reportaron una amplia incidencia de estas lesiones en pacientes mujeres y estas ocurrían entre la tercera a séptima década de vida ${ }^{(6)}$.

James J., estudió la prevalencia de lesiones exofíticas en una población americana, encontrando que el fibroma traumático representaba el $74.5 \%$ de todas las lesiones fibrosas exofíticas con un rango de prevalencia de 12/1000 personas mayores de 35 años. El $71 \%$ de estas lesiones se observaron en lengua y labio. Además, encontró que los fibromas traumáticos en el paladar duro, eran provocados en su mayoría por prótesis mal adaptadas (7).
Otro estudio revela que el fibroma traumático tiene un $69 \%$ de predilección por el sexo femenino y se encuentra en la segunda y sexta década de vida(8)

En una investigación que realizó Barzergar en el 2016, encontró una alta incidencia de fibroma traumático en pacientes diabéticos. Él llega a la conclusión de que esto se ve influenciado por los cambios en la condición ambiental oral, la perdida de algunos o todos los dientes y el uso de prótesis por parte de estos pacientes, el hábito de fumar, la ingesta de alcohol, la cicatrización retardada y la duración de la diabetes ${ }^{(9)}$.

En Perú el fibroma traumático tiene una prevalencia del $6.88 \%$ en relación a las lesiones bucomaxilofaciales y un $28.06 \%$ cuando se trata solamente de lesiones reactivas ${ }^{(10)}$.

Se encuentra generalmente en la mucosa del carrillo $(29 \%)$ (Figura 1) seguido de la mucosa labial (21.82\%) (Figura 2). Por lo general, se presenta como un crecimiento único y rara vez mide más de 1,5 centímetros. Otras áreas donde se puede encontrar son en la cara dorsal de la lengua (20\%) (Figura 3) y con menos frecuencia en el paladar duro (5.45\%)6 (Figura 4).

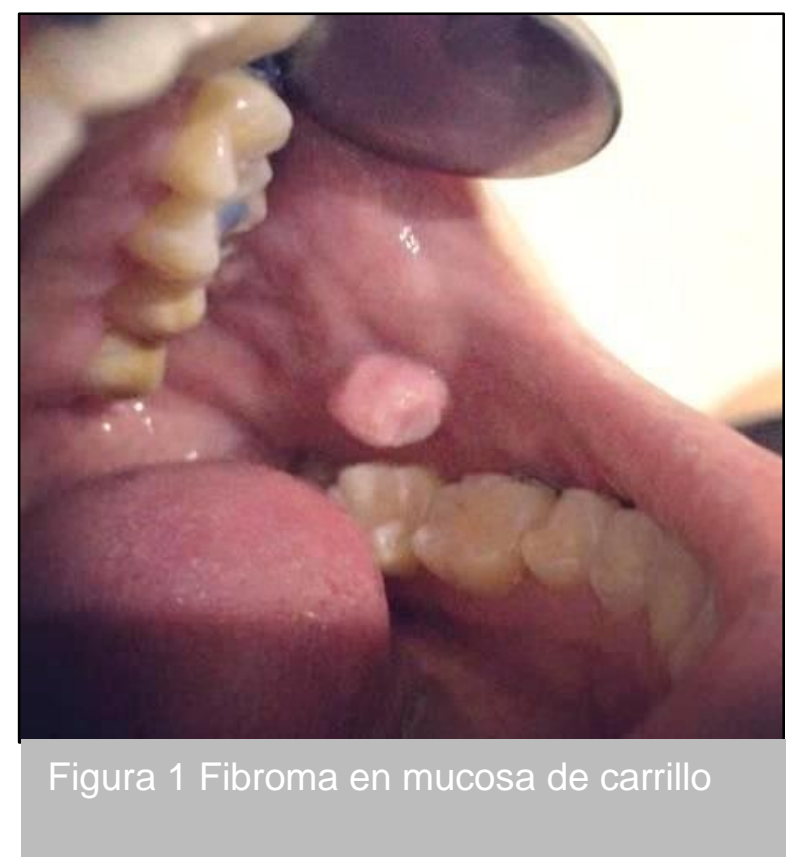



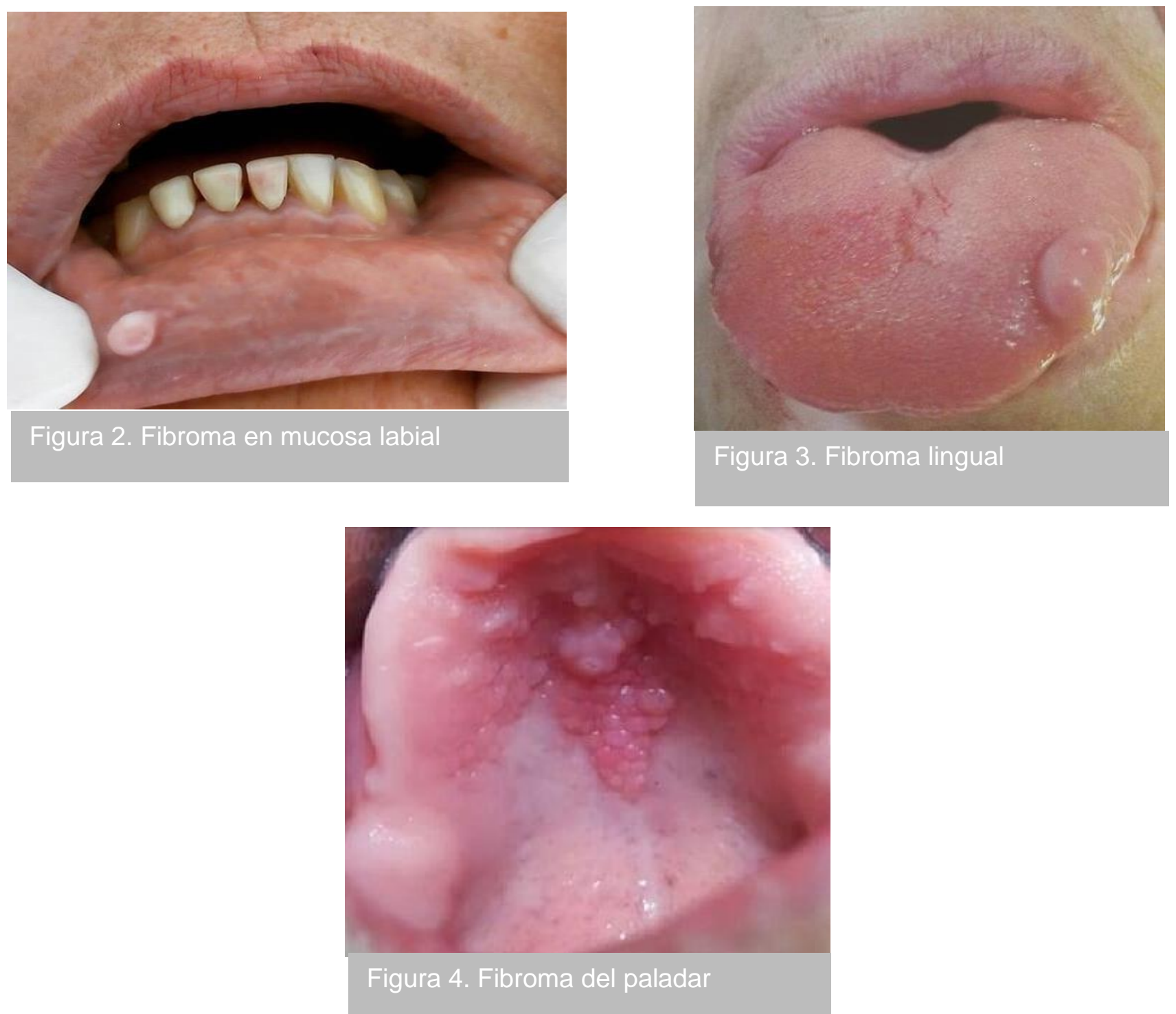

\section{METODOLOGÍA}

La revisión se basó fundamentalmente en la búsqueda de información en revistas indexadas a PubMed, NBCl y Scielo, con la utilización de descriptores como "fibroma traumático y causas", "fibroma inflamatorio y prevalencia". Fueron tenidos en cuenta los siguientes criterios.

Criterios de inclusión: Idiomas español, inglés; cualquier área geográfica o país; publicado desde el 2000 hasta el 2017. También fueron consultadas las tesis de grado científico, maestría y especialidades en el país, relacionadas con el tema. El análisis se centró en la exposición y síntesis de los resultados de los artículos revisados.

\section{ETIOLOGÍA}

La literatura en general ha encontrado que tanto el fibroma traumático así como el mucocele son consecuencia de hábitos orales. Esto se debe generalmente a una irritación crónica, como por ejemplo el morderse las mucosas (carrillo, labio o 
lengua), irritación por una cúspide de un diente y/o prótesis dentales. El uso de piercings linguales y labiales también ha sido asociado con el fibroma traumático (11).

Se encontró en un bebe de cuatro meses y medio un fibroma traumático asociado a un diente deciduo, esto indica claramente que la causa principal del fibroma traumático en cavidad oral son los irritantes locales ${ }^{(12)}$. Pueden ser de tamaño exagerado dependiendo del tiempo de la lesión, la intensidad de la irritación y los efectos metabólicos de las concentraciones séricas de hormonas, especialmente hormonas femeninas (13).

\section{Características clínicas}

El fibroma traumático se observa con más frecuencia en adultos que en niños. Ocurre entre la segunda y sexta década de vida y tiene una alta predilección por el sexo femenino. La incidencia disminuye después de la cuarta década de vida y esto se debe a la influencia de algunas hormonas en el desarrollo del fibroma traumático; el cual puede encontrarse en cualquier parte de la región oral. Sin embargo, se

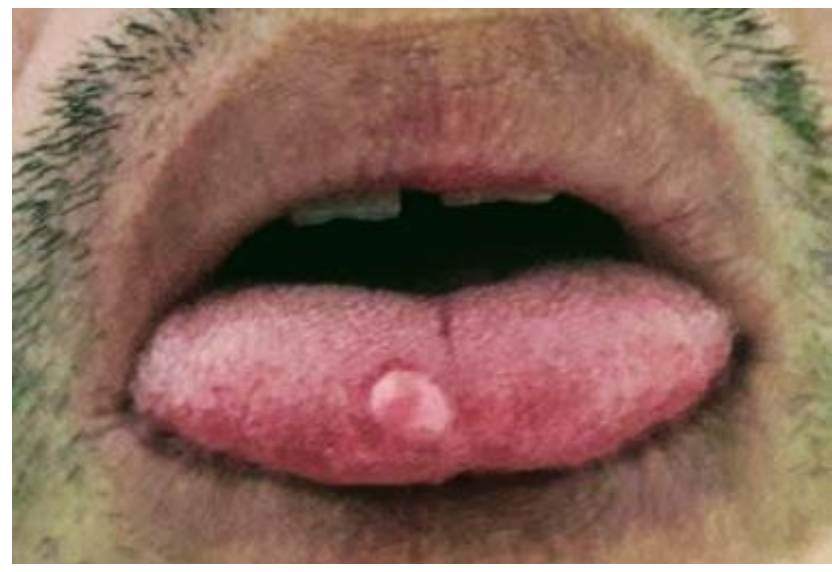

Figura 5. Fibroma en lengua

\section{Histopatología}

Desde el punto de vista histológico se presenta como una masa nodular sólida, no encapsulada, cubierta por epitelio escamoso estratificado, tejido conectivo denso con presencia de colágeno tipo I y III, dispuesto al azar y en ocasiones tejido conectivo fibroso hialinizado. Puede haber infiltrado inflamatorio crónico que contiene en su mayoría linfocitos y células plasmáticas ${ }^{(17) .}$ presenta con mayor frecuencia en la mucosa bucal en relación con el plano oclusal de los dientes maxilares y mandibulares ${ }^{(14)}$.

Es una masa pedunculada o sésil, redonda u ovalada, de superficie lisa, asintomática, no encapsulada. Puede tener el aspecto de una hoja si se desarrolló debajo de una prótesis. El tamaño puede variar entre $1 \mathrm{~mm}$. a $2 \mathrm{~cm}$. (Figura 5 y 6 ). Los fibromas traumáticos pueden desarrollarse durante semanas o meses hasta alcanzar su tamaño máximo, generalmente 2 $\mathrm{cm}$. de diámetro aunque en ocasiones pueden ser un poco más grandes. Su superficie puede estar ulcerada o hiperqueratósica debido a un trauma constante. Tiene el mismo color que la mucosa bucal pero en ocasiones puede ser pálido o un color más oscuro. Frecuentemente se presenta como una lesión única ${ }^{(14)}$. Cuando se observan lesiones múltiples, se debe considerar su diagnóstico asociado a algunas enfermedades como esclerosis tuberosa, fibromatosis familiar, síndrome de Cowden e hiperplasia fibrosa papilar del paladar. Los fibromas orales no evolucionan a cáncer ${ }^{(15)}$.

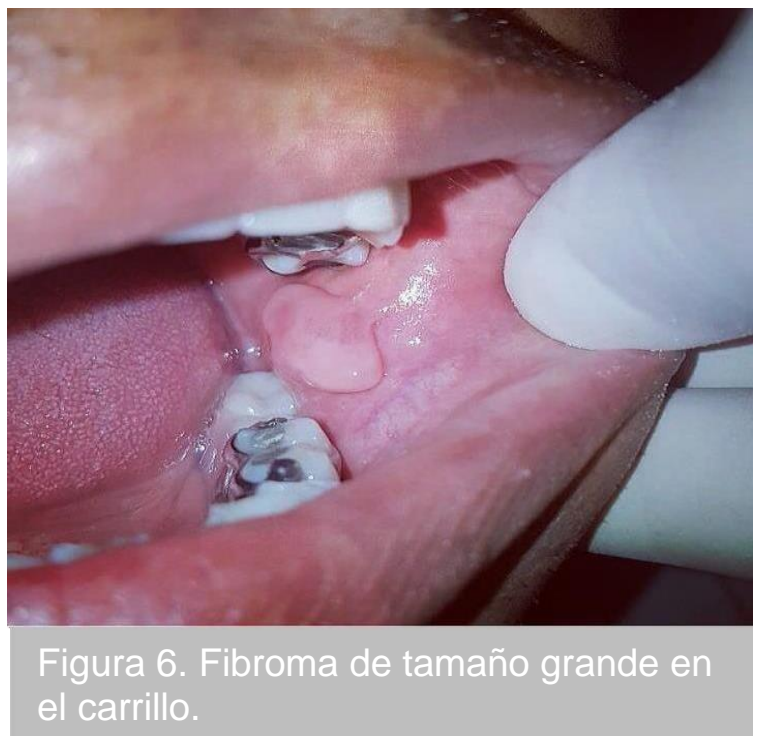

Para Kerr (18)., existen diversos tipos de fibras que se encuentran en los fibromas traumáticos:

- Fibras precolágenas: Son fibras de colágeno recién formadas y su presencia indica una el crecimiento activo del componente colágeno en el tejido conectivo fibroso. 
- Fibras elásticas: su presencia en los fibromas sugiere que se trata de un proceso inflamatorio o reparador y que ha pasado un periodo de tiempo considerable entre estas dos etapas.

- Fibras oxitalámicas: Su presencia puede sugerir un origen periodontal de las células de tejido conectivo que producen las fibras.

\section{Diagnóstico diferencial}

El diagnóstico diferencial de un fibroma traumático incluye neurofibromas, granuloma periférico de células gigantes, tumores de glándulas salivales benignos y malignos, granuloma piógeno, fibroma de células gigantes, granuloma osificante periférico, cáncer metastásico, hemangioma, inflamación gingival hiperplásica y angiosarcoma. Basado en su duración, el granuloma piógeno y el fibroma osificante periférico variarán de suave a firme y pueden sugerir un fibroma traumático, aunque son de color mucho más claro ${ }^{(19)}$. El fibroma traumático se asemeja al granuloma piógeno en el hecho de que ambos ocurren comúnmente en mujeres embarazadas, pero varía en el hecho de que el granuloma piógeno tiene un componente vascular mínimo y se presenta exclusivamente en la encía. Aunque los tumores metastásicos rara vez se observan en la región oral, el sitio comúnmente afectado es la encía adherida seguido por la lengua. Una lesión metastásica en la región oral, se encuentra en casi el $30 \%$ de los casos y las características histopatológicas deben parecerse al tumor de origen (20).

El fibroma traumático también se parece a ciertas lesiones como el mucocele (21). Los puntos clave para el diagnóstico diferencial son el color azulado, la palpación duro-elástica y la alta tasa de resolución espontánea en el caso del mucocele.

\section{Tratamiento}

En los primeros reportes sobre fibromas traumáticos, se informaba que cuando este era de tamaño pequeño y no causaba incomodidad al paciente no necesitaba ser retirado de boca. Sin embargo, si era muy grande, debía ser removido quirúrgicamente ${ }^{(22)}$.

Badcok J., decía, que para fibromas de tipo pedunculado se realiza la excisión y sutura. Por otro lado, en los fibromas de tipo sésil, se debe extirpar el periostio y la corteza del hueso al que están unidas estas lesiones ${ }^{(23)}$.

Actualmente, el tratamiento del fibroma traumático consiste en la escisión conservadora y quirúrgica de la lesión, a la vez, se debe eliminar el irritante que la causa.
La recidiva de estas lesiones es alta, por lo que se sugiere un seguimiento post operatorio.

El tejido extirpado debe enviarse para su respectivo examen microscópico y así determinar si se trata de un fibroma traumático o fibroma verdadero o una neoplasia benigna o maligna ${ }^{(24)}$. La eliminación del tejido puede hacerse utilizando bisturí manual o láseres de alta potencia como CO2 $(\lambda=10.6 \mu \mathrm{m})$, Er: YAG $(\lambda=2.94$ $\mu \mathrm{m})$, Er: YSGG $(\lambda=2.78 \mu \mathrm{m}), \mathrm{Nd}: \mathrm{YAG}(\lambda=1.64 \mu \mathrm{m}) \mathrm{y}$ láseres de diodo los cuales han demostrado varias ventajas en comparación con la técnica convencional, como por ejemplo una técnica más fácil, la reducción del tiempo de operación, la disminución del trauma en el tejido, la ausencia de sangrado durante el procedimiento (25).

La criocirugía es un procedimiento para el que se usa un líquido extremadamente frío o un instrumento que se llama criosonda para congelar y destruir el tejido anormal. Dentro de todos los procedimientos de criocirugía el nitrógeno líquido es el más usado debido a que es muy frío y versátil. Por la humedad y suavidad de la mucosa oral puede ser una primera opción de tratamiento, debido a que no es un procedimiento costoso, no produce sangrado y no provoca dolor en lesiones superficiales ${ }^{(26)}$.

La recurrencia de esta lesión no es frecuente. Sin embargo, Cooke reportó 3 casos de recidiva de 78 biopsias realizadas. El atribuyó que la razón de esto, fue que los factores causales que originaban los fibromas no fueron eliminados correctamente ${ }^{(27)}$.

\section{Conclusión}

El fibroma traumático es una lesión inocua y una afección común en la cavidad oral. Tiene predilección por el sexo femenino, entre la segunda y sexta década de vida. Se pueden tratar fácilmente mediante una historia clínica detallada, la biopsia por escisión quirúrgica y la evaluación histológica. Aunque es una entidad común, puede causar un dilema diagnóstico en un odontólogo sin experiencia. Se requiere de una correlación de hallazgos tanto clínicos como histopatológicos para tratar a estos pacientes. La eliminación de la fuente de irritación sigue siendo importante para evitar la recurrencia de las lesiones y se requiere un seguimiento post operatorio continúo teniendo en cuenta las posibilidades de recidiva. Por lo tanto, en este artículo, llegamos a la conclusión de que la prevención, el adecuado diagnóstico clínico e histológico patológico, el manejo y el tratamiento quirúrgico del fibroma traumático son importantes debido a su prevalencia y presentación semejante a crecimientos neoplásicos. 
Contribuciones de autoría: El autor recopiló la información y redactó el artículo.

Conflicto de interés: el autor declaró no tener conflicto de interés en esta publicación.

\section{REFERENCIAS}

1. Al-Rawi N. Localized reactive hyperplastic lesions of the gingiva: a clinico-pathological study of 636 lesions in Iraq. [Internet] J. Dent. Sci. 2009; 7 (1): 213-217.

2 Kolte, A.P. Focal fibrous overgrowths: A case series and review of literature. Contemp Clin Dent 2010; 1:271-274.

3. Harris J. Fibroma traumático riesgo potencial del tratamiento ortodóntico. CSV 2012; 4(1):132-137

4. Riya Verghese. Fibroma - A misnomer: Case Series. NUJHS 2015; 5(4): 83-87.

5. Jain $M$. True fibroma on the palate: $A$ unique case. "Int. J. Exp. Dent. Sci. 2016; 5(1): 69-71

6. Barker D. Localised fibrous overgrowths of the oral mucosa. Br J Oral Surg. 1967; 5(2):86-92.

7. James J. Oral exophytic lesions in 23,616 white Americans over 35 years of age. Oral Surg Oral Med Oral Pathol. 1986; 62(3):284-91.

8. Moret Y. Prevalencia de lesiones en la mucosa bucal de pacientes diagnosticados en el laboratorio central de histopatología bucal "Dr. Pedro Tinoco" de la Facultad de Odontología de la Universidad Central de Venezuela durante el periodo 19681987. Acta Odontol. Venez. 2007; 45(2): 1-7.

9. Barzegar A. Prevalence of Oral Lesions in Adult Diabetic Patients referred to Dentistry Faculty of Tabriz University of Medical Sciences between 2009-2014. Int.J.Curr.Microbiol.App.Sci 2016; 5(11): 906-911

10. Barria $\mathrm{H}$. Prevalencia de patolologías bucomaxilofaciales diagnosticadas histopatológicamente mediante biopsia en el servicio de estomatología quirúrgica del Hospital Nacional Arzobispo Loayza del 2010 al 2015. Tesis para obtener el título de Cirujano Dentista. Lima. Universidad San Martín de Porres; 2015. 111p.

11. Pradeed S. Inflammatory hyperplasia: From diagnosis to treatment. J Indian Soc Periodontol 2014 ; 18(1): 92-94.
12 Singh, S. Reactive fibrous hyperplasia associated with a natal tooth - A Case Report. J Indian SocPedoPrev Dent 2004; 22: 183-186.

13. Lanjekar A. An Unusually Large Irritation Fibroma Associated with Gingiva of Lower Left Posterior Teeth Region. Case Report. Dent. 2016.4p.

14. Das U. and Azher. Peripheral ossifying fibroma J Indian Soc Pedod Prev Dent.2009; 27(1):49.

15. Hunasgi S. Retrospective analysis of the clinical features of 530 cases of reactive lesions of oral cavity. J. adv. clin. res. insights. 2014; 1(1): 1-6.

16. Segura R. Oral manifestations of Cowden's disease. Presentation of a clinical case. Med Oral Patol Oral Cir Bucal 2006; 11:421-424.

17. Kashyap B. Reactive lesions of oral cavity: Asurvey of 100 cases in Eluru, West Godavari district. Contemp. Clin. Dent .2012; 3(3): 294- 297.

18. Oles RD. Incidence and distribution of various connective tissue fibers in oral fibromas. Oral Surg Oral Med Oral Pathol. 1968 Oct; 26(4):487-96.

19. Navnita S. Traumatic fibroma: A case series. J Res Health Sci 2016; 7(1): 28-31.

20. Pandey R. Pyogenic granuloma of buccal mucosa mimicking as traumatic fibroma in pregnancy. Indian J Dent Adv 2016; 8(3): 172-175.

21. Valerio R. Mucocele and fibroma: treatment and clinical features for differential diagnosis. Braz Dent J. 2013; 24(5):537-41.

22 Eversole LR, Rovin S. Reactive lesions of the gingiva. J Oral Pathol 1972; 1:30-8.

23. Badcock J. Oral bilateral symmetrical fibromas. Report of a case. Oral Surg Oral Med Oral Pathol. 1966; 21(1):4-8.

24. Anand A. Chhina S, Goel A, Mishra S, Kazimm J, SauravK. Irritational Fibroma: A Case Report. International Journal of Oral Health and Medical Research 2016; 2(5): 78-80.

25. Bakhtiari S. Removal of an Extra-large Irritation Fibroma with a Combination of Diode Laser and Scalpel. J Laser Med Sci. 2015; 6(4): 182-184.

26. Ishida C. Cryosurgery in oral lesions. Int J Dermatol 1998; 37: 283-285.

27. Cooke B. "The fibrous epulis and the fibroepithelial polyp: their histogenesis and natural history." $\mathrm{Br}$ Dent J. 1952. 93. 305-309. 